\title{
A sufficient condition on the existence of pure equilibrium in two-person symmetric zerosum games
}

Citation for published version (APA):

Ismail, M. S. (2014). A sufficient condition on the existence of pure equilibrium in two-person symmetric zerosum games. Maastricht University, Graduate School of Business and Economics. GSBE Research Memoranda No. 035 https://doi.org/10.26481/umagsb.2014035

Document status and date:

Published: 01/01/2014

DOI:

10.26481/umagsb.2014035

Document Version:

Publisher's PDF, also known as Version of record

\section{Please check the document version of this publication:}

- A submitted manuscript is the version of the article upon submission and before peer-review. There can be important differences between the submitted version and the official published version of record.

People interested in the research are advised to contact the author for the final version of the publication, or visit the DOI to the publisher's website.

- The final author version and the galley proof are versions of the publication after peer review.

- The final published version features the final layout of the paper including the volume, issue and page numbers.

Link to publication

\footnotetext{
General rights rights.

- You may freely distribute the URL identifying the publication in the public portal. please follow below link for the End User Agreement:

www.umlib.nl/taverne-license

Take down policy

If you believe that this document breaches copyright please contact us at:

repository@maastrichtuniversity.nl

providing details and we will investigate your claim.
}

Copyright and moral rights for the publications made accessible in the public portal are retained by the authors and/or other copyright owners and it is a condition of accessing publications that users recognise and abide by the legal requirements associated with these

- Users may download and print one copy of any publication from the public portal for the purpose of private study or research.

- You may not further distribute the material or use it for any profit-making activity or commercial gain

If the publication is distributed under the terms of Article $25 \mathrm{fa}$ of the Dutch Copyright Act, indicated by the "Taverne" license above, 
Mehmet Ismail

A sufficient condition on the existence of pure equilibrium in two-person symmetric zerosum games

$\mathrm{RM} / 14 / 035$

\section{GSBE}

Maastricht University School of Business and Economics

Graduate School of Business and Economics

P.O Box 616

NL- 6200 MD Maastricht

The Netherlands 


\title{
A sufficient condition on the existence of pure equilibrium in two-person symmetric zerosum games*
}

\author{
Mehmet ISMAIL ${ }^{\dagger}$
}

October, 2014

\begin{abstract}
In this note, we introduce a new sufficient condition, called signquasiconcavity, on the existence of a pure equilibrium in two-person symmetric zerosum games, which generalizes both generalized ordinal potentials (Monderer and Shapley, 1996) and quasiconcavity (Duersch et al., 2012).

Keywords: Symmetric zerosum games, Pure equilibrium, Quasiconcave games, Generalized ordinal potential games

JEL Classification: C72
\end{abstract}

\section{Introduction}

In Ismail (2014) we show that there is an equivalence between the class of two-person symmetric zerosum games and the class of decision problems with a complete (not necessarily transitive) preference relation. Accordingly, we show that a strategy is a pure optimal strategy in a two-person symmetric

*I am indebted to Ronald Peeters for his comments and suggestions. I would like to thank Jean-Jacques Herings for his feedback on an earlier version. I am thankful to the audiences at Copenhagen University, SING9 conference at the University of Vigo and in a poster presentation at the 24th Jerusalem School in Economic Theory, where part of this work has been presented. Of course, any mistake is mine.

$\dagger^{\dagger}$ Department of Economics, Maastricht University, The Netherlands. E-mail: mehmet@mehmetismail.com 


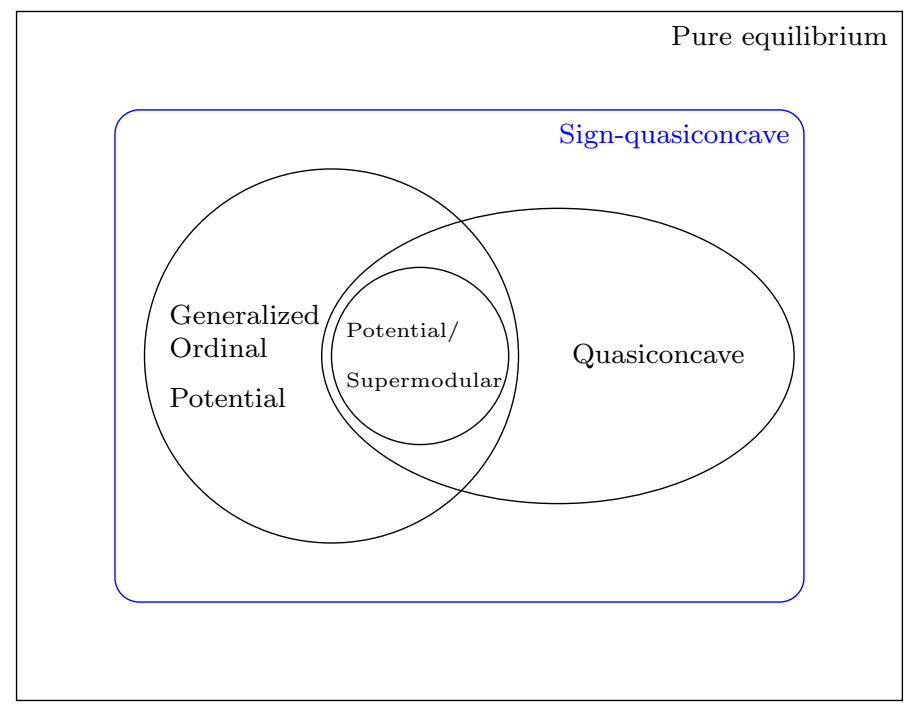

Figure 1: Relations between sufficient conditions on pure equilibria in twoperson symmetric zerosum games.

zerosum game if and only if it is a maximal element in its equivalent decision problem. By exploiting this equivalence, we find a sufficient condition, called sign-quasiconcavity, for the the existence of a pure equilibrium which generalizes both generalized ordinal potentials (Monderer and Shapley, 1996) and quasiconcavity (Duersch et al., 2012).

A game is called sign-quasiconcave if it is quasiconcave with respect to the sign of the payoff function. We show that if a two-person symmetric zerosum game is sign-quasiconcave then it possesses an equilibrium in pure strategies. We provide an example of a game that is sign-quasiconcave but neither has it generalized ordinal potential nor it is quasiconcave. In Figure 1, the inclusion relationships between several properties in two-person symmetric zerosum games is shown. ${ }^{1}$ Monderer and Shapley (1996) showed that a potential game is a generalized ordinal potential game and that these games possess pure equilibrium. Duersch et al. (2012) showed that if a two-person symmetric zerosum game is quasiconcave then it has a pure equilibrium. Moreover, they showed that potential games and supermodular games (Topkis, 1998) coincide in two-person symmetric zerosum games. We provide an example to show that generalized ordinal potential games are not a special case of

\footnotetext{
${ }^{1}$ Note that the sizes of the shapes in Figure 1 is drawn arbitrarily.
} 
quasiconcave games. A potential game, however, is always a quasiconcave game but the converse is not true as it is shown in Duersch et al. (2012, p. 559).

\section{The framework}

A two-person symmetric zerosum game can be denoted by a pair $(X, v)$ where both players have the same set of pure actions $X$ and $v: X \times X \rightarrow \mathbb{R}$ is the utility function of, say, player 1 . The utility function of player 2 is the transpose of $v$ satisfying $v(x, y)=-v^{\top}(x, y)$ for all $x, y$ in $X$.

We denote a decision problem by the pair $(X, \succeq)$ where $X$ is the set of alternatives and the relation $\succeq \subseteq X \times X$ represents the preferences of the decision maker on $X$ and it is assumed to be complete but not necessarily transitive. A decision problem can be interpreted as if a decision maker is to choose an element taking into account her preferences. Since we do not assume that preferences are transitive it is not in general possible to represent the preferences by a one-variable order-preserving utility function. In that case, a convenient approach to obtain a representation is to consider a twovariable utility function. ${ }^{2}$ We say that a real-valued function $v$ represents the relation $\succeq$ if for all $x$ and $y$ in $X$ we have $v(x, y)>0$ if and only if $x \succ y$.

In addition, if the function $v$ represents the preference relation $\succeq$ on $X$ then we call the pair $(X, \succeq)$ the equivalent decision problem of the two-person symmetric zerosum game $(X, v)$. The equivalent game of a decision problem may be interpreted as the decision maker is playing against her dual-self where the gain of the decision maker is the loss (since the game is zerosum) of her dual-self. ${ }^{3}$

Proposition 1. (Ismail, 2014). Let $(X, v)$ be a two-person symmetric zerosum game and let $(X, \succeq)$ be its equivalent decision problem. $X$ admits a maximal element $x^{*}$ with respect to $\succeq$ if and only if the game $(X, v)$ possesses a pure equilibrium $\left(x^{*}, x^{*}\right)$.

This result is useful for deriving sufficient conditions for the existence of a pure equilibrium in two-person symmetric zerosum games. The idea is to use a basic structure of the preference relations such as acyclicity and transitivity

\footnotetext{
${ }^{2}$ Fishburn (1982) is probably one of the most well-known supporter of this approach.

${ }^{3}$ See, for example, Fudenberg and Levine (2006) for a similar interpretation of decision problems.
} 
to obtain a sufficiency result for two-person symmetric zerosum games. Let us define acyclicity formally.

Definition 1. A preference relation $\succeq$ on a set $X$ is said to be acyclic if for all finite subsets $\left\{x_{1}, x_{2}, \ldots, x_{n}\right\} \subset X$,

$$
x_{1} \succ x_{2}, x_{2} \succ x_{3}, \ldots, x_{n-1} \succ x_{n} \text { implies } x_{1} \succeq x_{n} \text {. }
$$

\section{Sufficient conditions}

The question 'how far we can go?' is not an unimportant one for sufficiency investigations. The following proposition makes use of a quite general sufficient condition for the existence of a maximal element in order to draw a conclusion in two-person symmetric zerosum games.

Proposition 2. Let $(X, v)$ be a two-person symmetric zerosum game where $X$ is a compact set in a topological space and let $(X, \succeq)$ be its equivalent decision problem. If the relation $\succeq$ is acyclic such that for all $x \in X$ the set $\{y \in X \mid x \succ y\}$ is open in the relative topology of $X$. Then the game $(X, v)$ possesses a pure equilibrium.

Proof. If the hypotheses of the proposition are satisfied and if the preference relation $\succeq$ is acyclic then $X$ admits a maximal element $x^{*} \in X$ with respect to $\succeq$ by Bergstrom (1975). ${ }^{4}$ Hence by Proposition 1 , the game $(X, v)$ possesses a pure equilibrium.

Corollary 1. Let $(X, v)$ be a finite two-person symmetric zerosum game whose equivalent decision problem is $(X, \succeq)$. If the relation $\succeq$ is acyclic then $(X, v)$ possesses a pure equilibrium.

Remark. It is well known that if a relation is transitive then it is acyclic. Therefore assuming transitivity of $\succeq$ instead of acyclicity does not change the conclusion in Corollary 1.

Quasiconcave games were introduced by Radzik (1991) and may be interpreted as a 'discrete version' of quasiconcavity of payoff functions. In two-person symmetric zerosum games, if each column has a 'single peak'

\footnotetext{
${ }^{4}$ Although there are more general sufficient conditions on the existence of a maximal element in the literature, we use this one due to its simplicity. Note also that this result has been independently discovered by several authors. See Walker (1977) for a discussion.
} 
then we call the game quasiconcave. By exploiting the symmetry feature, Duersch et al. (2012) showed that if a two-person symmetric zerosum game is quasiconcave then it has a pure equilibrium. Now let us define a weaker version of quasiconcavity called sign-quasiconcavity.

Definition 2. A symmetric two-person zerosum game $(X, v)$ is said to be sign-quasiconcave if there exists a total order $>$ on $X$ such that for every $x, y, z$ and every $w$ in $X$ with $x>y>z$ then

$$
\operatorname{sgn}(v(y, w)) \geq \min \{\operatorname{sgn}(v(x, w)), \operatorname{sgn}(v(z, w))\} .
$$

To put it differently, the game $(X, v)$ is sign-quasiconcave if and only if the game $(X, \operatorname{sgn}(v))$ is quasiconcave. The following proposition shows the relationship between quasiconcavity and sign-quasiconcavity.

Proposition 3. If a two-person symmetric zerosum game is quasiconcave then it is sign-quasiconcave.

Proof. By contraposition assume that $(X, v)$ is not sign-quasiconcave then for every total order $>$ there exists $x, y, z$ and $w$ in $X$ with $x>y>z$ such that $\operatorname{sgn}(v(y, w))<\operatorname{sgn}(v(x, w))$ and $\operatorname{sgn}(v(y, w))<\operatorname{sgn}(v(z, w))$. There are six ways that can occur: $\operatorname{sgn}(v(y, w))$ can be either 0 or $-1, \operatorname{sgn}(v(x, w))$ and $\operatorname{sgn}(v(z, w))$ can be either 0 or 1 . If $\operatorname{sgn}(v(x, w))=1, \operatorname{sgn}(v(y, w))=0$ and $\operatorname{sgn}(v(z, w))=1$ then $v(y, w)=0, v(x, w)>0$ and $v(z, w)>0$ hence $(X, v)$ is not quasiconcave. The other cases are very similar, so quasiconcavity implies sign-quasiconcavity.

It turns out that quasiconcavity is not necessary for sign-quasiconcavity. For a counter-example see the game in the Figure 2. The following proposition establishes a connection between sign-quasiconcave games and their equivalent decision problems.

Lemma 1. Let $(X, v)$ be a two-person symmetric zerosum game. If it is sign-quasiconcave, then the relation $\succeq$ is acyclic in the equivalent decision problem $(X, \succeq)$ of the game $(X, v)$.

Proof. Firstly, notice that the equivalent decision problem $(X, \succeq)$ of $(X, v)$ and $(X, \operatorname{sgn}(v))$ are the same because for every $x, y$ in $X, v(x, y)>0$ if and only if $\operatorname{sgn}(v(x, y))>0$.

By contraposition, we will show that if $\succeq$ on $X$ is cyclic then the game $(X, v)$ is not sign-quasiconcave. Assume $\succeq$ is cyclic then there exists a finite 
subset $\bar{X} \subseteq X$ that does not contain a maximal element. So by Proposition 3 of Ismail (2014), the two-person symmetric zerosum game $\left(\bar{X}, \operatorname{sgn}\left(v_{\mid \bar{X}}\right)\right)^{5}$ has no pure equilibrium. Then by Theorem 1 in Duersch et al. (2012), the game $\left(\bar{X}, \operatorname{sgn}\left(v_{\mid \bar{X}}\right)\right)$ is not quasiconcave. If it is not possible to find a total order on $\bar{X}$ such that the game $\left(\bar{X}, \operatorname{sgn}\left(v_{\mid \bar{X}}\right)\right)$ is not quasiconcave then it is not possible to find a total order on $X$ such that the larger game $(X, \operatorname{sgn}(v))$ is quasiconcave. Hence $(X, \operatorname{sgn}(v))$ is not quasiconcave.

Theorem 1. If a finite two-person symmetric zerosum game $(X, v)$ is signquasiconcave then it possesses a pure equilibrium.

Proof. By Lemma 1 sign-quasiconcavity of $(X, v)$ implies acyclicity of $\succeq$ in the equivalent decision problem. Then Corollary 1 implies that $(X, v)$ has a pure equilibrium.

Since we showed that sign-quasiconcavity is a weaker condition than quasiconcavity in Propoisition 3, Theorem 1 generalizes Theorem 1 of Duersch et al. (2012) where they show that every finite two-person symmetric zerosum quasiconcave game has a pure equilibrium.

The notion of generalized ordinal potential game is introduced by Monderer and Shapley (1996). It is a sufficient condition for the existence of a pure equilibrium in normal-form games. Here we are interested in generalized ordinal potentials in a specific class, namely in two-person symmetric zerosum games. Let us now formally define it for this class.

Definition 3. A symmetric two-person zerosum game $(X, v)$ is a generalized ordinal potential game if there exists a function $P: X \times X \rightarrow \mathbb{R}$ such that for all $y$ and all $x, z$ in $X,{ }^{6}$

$$
\begin{aligned}
& v(x, y)-v(z, y)>0 \text { implies } P(x, y)-P(z, y)>0 \\
& v(x, y)-v(z, y)>0 \text { implies } P(y, x)-P(y, z)>0 .
\end{aligned}
$$

The following proposition illustrates that the equivalent decision problem of a generalized ordinal potential game is transitive.

\footnotetext{
${ }^{5}$ The notation $v_{\mid \bar{X}}$ denotes the function $v$ restricted to the domain $\bar{X}$.

${ }^{6}$ Note that the second line below is expressed in terms of the payoff function of player 1 instead of player 2 .
} 
Proposition 4. Let $(X, v)$ be a two-person symmetric zerosum game and let $(X, \succeq)$ be its equivalent decision problem. If $(X, v)$ has a generalized ordinal potential, then $\succeq$ is transitive and for all $x, y$ and $z$ in $X$ with $x \succeq y \succeq z$,

$$
v(x, z) \geq \min \{v(x, y), v(y, z)\} .
$$

Proof. By contraposition, we will show in three cases that if $\succeq$ is not transitive then $(X, v)$ has no generalized ordinal potential.

Case 1: Suppose there exists $x, y, z$ in $X$ with $x \succ y \succ z \succ x$. Then the decision problem $(\{x, y, z\}, \succeq)$ has no maximal element which implies the game $\left(\{x, y, z\}, v_{\mid\{x, y, z\}}\right)$ associated to it has no pure equilibrium by Proposition 3 of Ismail (2014). Therefore, it does not have a generalized ordinal potential by Monderer and Shapley (1996, p. 128-129). Since $\left(\{x, y, z\}, v_{\mid\{x, y, z\}}\right)$ has no generalized ordinal potential, the game $(X, v)$ does not have too. ${ }^{7}$

Case 2: Suppose there are $x, y$ and $z$ in $X$ with $x \succ y \succ z \sim x$. It implies $v(x, y)>0, v(y, z)>0$ and $v(x, z)=0$. Accordingly, a potential function must satisfy the following impossible sequence of relations: $P(z, x)>P(y, x)>P(y, z)>P(x, z)>P(x, y)>P(z, y)>P(z, x)$.

Case 3: Suppose there exists $x, y$ and $z$ in $X$ with $x \succ y \sim z \sim x$ which implies $v(x, y)>0, v(y, z)=0$ and $v(x, z)=0$. It further implies that a potential function must satisfy $P(z, x)>P(y, x)>P(y, z)=P(x, z)>$ $P(x, y)>P(z, y)=P(z, x)$ which leads to a contradiction again.

Finally, assume by contradiction that there exists $x \succeq y \succeq z$ such that $v(x, z)<\min \{v(x, y), v(y, z)\}$. Then a potential function for the game $(X, v)$ fails to satisfy the following sequence of relations: $P(z, x)>P(y, x)>$ $P(y, z)>P(x, z)>P(x, y)>P(z, y)>P(z, x)$.

It is an open question whether the conditions given in the Proposition 4 are sufficient for the existence of a generalized ordinal potential. When we require the game to be an exact potential game ${ }^{8}$, however, we are able to characterize the game with respect to its equivalent decision problem. As it might be expected due to a stronger requirement of exact potential games, the equivalent decision problems additionally satisfy the independence axiom. Ismail (2014) shows that a two-person symmetric game is an exact potential

\footnotetext{
${ }^{7}$ Clearly this is true for any game. Because if it is not possible to construct a generalized ordinal potential for $v_{\mid \bar{X}}$ where $\bar{X} \subseteq X$ then it is not possible to construct one for $v$.

${ }^{8}$ The definition of exact potential game can be obtained when we modify Definition 3 as follows. We replace strict inequalities with an equality and replace 'implies' parts with 'if and only if'.
} 
game if and only if its equivalent decision problem satisfies von NeumannMorgenstern utility. The following proposition illustrates that transitivity implies sign-quasiconcavity in finite games.

Proposition 5. Suppose that $\succeq$ is transitive in the equivalent decision problem $(X, \succeq)$ of a finite two-person symmetric zerosum game $(X, v)$. Then, the game $(X, v)$ is sign-quasiconcave.

Proof. Take an element in $X$ which is minimal with respect to $\succeq$ and call it $x_{1}$. Take a minimal element from $X \backslash\left\{x_{1}\right\}$ and call it $x_{2}$. Then take a minimal element in $X \backslash\left\{x_{1}, x_{2}\right\}$ and call it $x_{3}$. Continue the process until no element remains in $X$. Define the total order $>$ on $X$ as follows: We have $x_{j}>x_{i}$ whenever $j>i$. Note also that we have defined $>$ such that $j>i$ only if $x_{j} \succeq x_{i}$. We show that for every $x_{k}, x_{j}, x_{i}$ and every $x_{m}$ in $X$ with $x_{k}>x_{j}>x_{i}$ we have $\operatorname{sgn}\left(v\left(x_{j}, x_{m}\right)\right) \geq \min \left\{\operatorname{sgn}\left(v\left(x_{k}, x_{m}\right)\right), \operatorname{sgn}\left(v\left(x_{i}, x_{m}\right)\right)\right\}$. There are two cases to consider.

Case 1: Suppose $x_{m}>x_{j}$ which implies that $x_{m} \succeq x_{k}$ hence we have $\operatorname{sgn}\left(v\left(x_{j}, x_{m}\right)\right) \leq 0$. Case 1.1: Suppose that $v\left(x_{j}, x_{m}\right)=0$. Since $x_{m} \succeq x_{i}$ we have $\operatorname{sgn}\left(v\left(x_{i}, x_{m}\right)\right) \leq 0$. Case 1.2: Suppose that $\operatorname{sgn}\left(v\left(x_{j}, x_{m}\right)\right)=-1$ which means $x_{m} \succ x_{j}$. By transitivity, it implies $x_{m} \succ x_{i}$, so we have $\operatorname{sgn}\left(v\left(x_{i}, x_{m}\right)\right)=-1$.

Case 2: Suppose $x_{j}>x_{m}$ which implies that $x_{j} \succeq x_{m}$ hence we have $\operatorname{sgn}\left(v\left(x_{j}, x_{m}\right)\right) \geq 0$. Case 2.1: Suppose that $v\left(x_{j}, x_{m}\right)=0$. Since $x_{m} \succeq x_{i}$, $\operatorname{sgn}\left(v\left(x_{i}, x_{m}\right)\right) \leq 0$. Case 2.2: If $\operatorname{sgn}\left(v\left(x_{j}, x_{m}\right)\right)=1$ then the inequality is satisfied because it is the largest value of the sign function.

Corollary 2. If a two-person symmetric zerosum game has a generalized ordinal potential, then it is sign-quasiconcave.

Proof. By Proposition 4, if a game two-person symmetric zerosum game has a generalized ordinal potential, then the relation $\succeq$ is transitive in its equivalent decision problem. Hence Proposition 5 applies.

Let us now illustrate some of the results we have been discussing. Consider the two-person symmetric zerosum game $(X, v)$ in Figure 2. Only player 1's payoff matrix is given. The rows and columns are labeled by the actions $x, y, z$ and $w$. Notice that the payoff function of the game $(X, \operatorname{sgn}(v))$ is the sign function of $v$ and that the place of the actions has been changed with respect to the game on the left. 


$\begin{aligned} & x \\ & y \\ & z \\ & w\end{aligned}\left(\begin{array}{cccc}0 & -3 & -1 & -5 \\ 3 & 0 & 6 & 3 \\ 1 & -6 & 0 & 2 \\ 5 & -3 & -2 & 0\end{array}\right), \quad \begin{aligned} & y \\ & w \\ & x\end{aligned}\left(\begin{array}{cccc}0 & 1 & 1 & 1 \\ -1 & 0 & 1 & 1 \\ -1 & -1 & 0 & 1 \\ -1 & -1 & -1 & 0\end{array}\right)$

Figure 2: Games $(X, v)$ and $(X, \operatorname{sgn}(v))$ respectively.

Observe that the strategy $y$ guarantees the payoff of zero (the value of the game) so it is an optimal strategy and $(y, y)$ is a pure equilibrium in both games. To the best of our knowledge, however, the sufficient conditions that is known in the literature cannot predict that this game has a pure strategy equilibrium. By contrast, this game is sign-quasiconcave. To see this, we consider the sign function of $v$ and order the actions as $y, z, w, x$ which is given on the right in Figure 2. Notice that in this game each column has a single peak, therefore making the game $(X, v)$ sign-quasiconcave. By Theorem 1 we conclude that $(X, v)$ has a pure equilibrium.

Firstly, notice that the game $(X, v)$ is not quasiconcave, because, no matter how you order the actions, it is not possible to obtain a matrix such that each column has a single-peak. Secondly, let $(X, \succeq)$ be the equivalent decision problem of $(X, v)$. Since we have $z \succ w \succ x$ and $v(z, x)<$ $\min \{v(z, w), v(w, x)\}$ by Proposition 4 the game $(X, v)$ cannot have a generalized ordinal potential.

It follows by definition that sign-quasiconcavity is invariant under multiplication of a particular payoff by a positive constant. For example, let us multiply $v(w, x)$ by $\frac{1}{5}$ which equals 1 and set $v(x, w)=-1$ keeping the symmetry property of the game in Figure 2 . Then the game $(X, v)$ would have a generalized ordinal potential but would still fails to be quasiconcave. The generalized ordinal potential function is given by: $P(x, x)=1, P(x, y)=$ $14, P(x, z)=6, P(x, w)=3, P(x, x)=15, P(y, y)=16, P(y, z)=12, P(y, w)$ $=13, P(z, x)=8, P(z, y)=10, P(z, z)=9, P(z, w)=7, P(w, x)=2, P(w, y)$ $=11, P(w, z)=5$ and $P(w, w)=4$.

\section{Conclusion}

In this note, we introduced a property of games called sign-quasiconcavity. We showed that two-person symmetric zerosum games possessing this property has at least one equilibrium in pure strategies, which generalizes both 
generalized ordinal potentials (Monderer and Shapley, 1996) and quasiconcavity (Duersch et al., 2012). In addition, these inclusions are strict as shown in Figure 2.

\section{References}

Bergstrom, T. C. (1975). Maximal elements of acyclic relations on compact sets. Journal of Economic Theory 10(3), 403 - 404.

Duersch, P., J. Oechssler, and B. C. Schipper (2012). Pure strategy equilibria in symmetric two-player zero-sum games. International Journal of Game Theory 41, 553-564.

Fishburn, P. C. (1982). Nontransitive measurable utility. Journal of Mathematical Psychology 26(1), 31-67.

Fudenberg, D. and D. K. Levine (2006). A dual-self model of impulse control. The American Economic Review 96(5), pp. 1449-1476.

Ismail, M. (2014). The equivalence between two-person symmetric games and decision problems. GSBE Research Memorandum 14/023.

Monderer, D. and L. S. Shapley (1996). Potential games. Games and Economic Behavior 14(1), 124-143.

Radzik, T. (1991). Saddle point theorems. International Journal of Game Theory 20, 23-32.

Topkis, D. (1998). Supermodularity and Complementarity. Princeton University Press.

von Neumann, J. (1928). Zur Theorie der Gesellschaftsspiele. Mathematische Annalen 100, 295-320.

Walker, M. (1977). On the existence of maximal elements. Journal of Economic Theory, 470-474. 